\title{
Embryonic Development of Axon Pathways in the Drosophila CNS. II. Behavior of Pioneer Growth Cones
}

\author{
J. Roger Jacobs and Corey S. Goodman \\ Department of Biochemistry, Howard Hughes Medical Institute, University of California, Berkeley, Berkeley, California \\ 94720
}

\begin{abstract}
We have identified the neurons that pioneer the major CNS axon tracts in the Drosophila embryo and determined their trajectory and fasciculation choices using serial section electron microscopy. Although Drosophila pioneer neurons make choices similar to those of their grasshopper homologs, there are interesting differences that reflect the much smaller nervous system size and the much faster rate of development characteristic of Drosophila. For example, where 2 longitudinal tracts are pioneered independently in grasshopper, only one is formed in Drosophila. This change is due to a change in fasciculation affinity of the $\mathrm{PCC}$ growth cone. Additionally, the intersegmental (IS) nerve is pioneered by a different neuron in Drosophila (aCC) than in the grasshopper (U1) because the smaller Drosophila CNS places the IS nerve within filopodial reach of the aCC soma, while in the grasshopper it is not.

Drosophila growth cones explore a much more confined neuropil volume than do grasshopper growth cones but can also sample a larger fraction of the CNS as well. For this reason, some cell-cell recognition events critical to pathfinding in the grasshopper embryo may not be as essential in Drosophila. Nevertheless, many specific cellular affinities have been retained through the evolutionary divergence of these 2 species.
\end{abstract}

Great strides have been made in our understanding at the cellular level of how growth cones find their correct pathways during neuronal development. Much of this progress has come from studies on identified neurons in the relatively simple nervous systems of such diverse organisms as nematodes, insects, and fish. These studies have revealed that, among other guidance mechanisms, growth cones display selective affinities for particular axonal surfaces that lead to stereotyped sequences of specific pathway choices.

For example, in the grasshopper, the growth cone of the G neuron turns anteriorly on the A/P axon fascicle only if it contacts the $\mathrm{P}$ axons (Bastiani et al., 1984); similarly, the pCC growth cone extends anteriorly only if it contacts the MPl and dMP2 axons (Bastiani et al., 1986). In the Japanese medaka fish, the DL1 growth cone extends in the dorsolateral fascicle

Received Sept. 20, 1988; revised Nov. 29, 1988; accepted Dec. 5, 1988

We thank Michael Bastiani for Figure $11 \mathrm{~A}$, Frances Thomas for technical assistance, and John Stevens for the use of his serial reconstruction equipment. This work was supported by a Canadian MRC Fellowship (J.R.J.) and the Howard Hughes Medical Institute and NIH Grant HD21294 (C.S.G.).

Correspondence should be addressed to J. Roger Jacobs at the above address.

Copyright (C) 1989 Society for Neuroscience $0270-6474 / 89 / 072412-11 \$ 02.00 / 0$ of the spinal cord only if it contacts the Rohon Beard axons (Kuwada, 1986). And in the nematode, extension of the AVM axon into the circumesophageal ring requires contact with the BUDL axon (Walthall and Chalfie, 1988). In all 4 examples, proper projection of individual axons can be prevented by experimental removal of the specific axons with which they normally fasciculate.

We would like to study the mechanisms of these events at the molecular genetic level in order to determine how the specificity of neuron-neuron recognition is established. Although much of the previous cellular analysis of growth cone guidance in the insect CNS has been done on the grasshopper embryo, the power of molecular genctic approaches using Drosophila makes it important to understand the development of the developing fly CNS at the same level of resolution.

Grasshopper and Drosophila have also been extensively used for studies on growth cone guidance in the PNS. The grasshopper studies on growth cone guidance in the PNS have focused on sensory neurons in appendages such as limb buds (e.g., Caudy and Bentley, 1986a, b). The Drosophila studies have focused on sensory neurons in the imaginal discs that metamorphose into appendages such as wings and legs (e.g., Blair and Palka, 1985; Jan et al., 1985; Blair et al., 1987), as well as on sensory neurons in the head and body segments of the developing embryo (e.g., Ghysen et al., 1986; Bodmer and Jan, 1987; Steller et al., 1987).

In this paper we examine the pathway choices, cell interactions, and patterns of selective fasciculation of the initial growth cones in the Drosophila CNS during the formation of the major axon tracts. The major players and their behavior are very similar between the grasshopper and Drosophila (Thomas et al., 1984; Bastiani et al., 1985), but there are interesting speciesspecific differences in the relative timing and sequence of cellcell interactions, as well as certain axon fasciculation choices, which imply that some cell-cell recognition events have changed during evolution, in some cases by either the acquisition or deletion of specific cellular affinities.

\section{Materials and Methods}

All methods used are as described in the preceding paper (Jacobs and Goodman, 1989).

\section{Results}

Embryogenesis in the fruit fly Drosophila melanogaster requires $22 \mathrm{hr}$ until hatching at $25^{\circ} \mathrm{C}$. Progenitors of the CNS, the neuroblasts, delaminate from the ventral ectoderm beginning about $4 \mathrm{hr}$ of development. Over the next $8 \mathrm{hr}$ or so, the neuroblasts segregate, divide, and produce a stereotyped lineage of ganglion mother cells, which, in turn, each divide once to generate pairs 

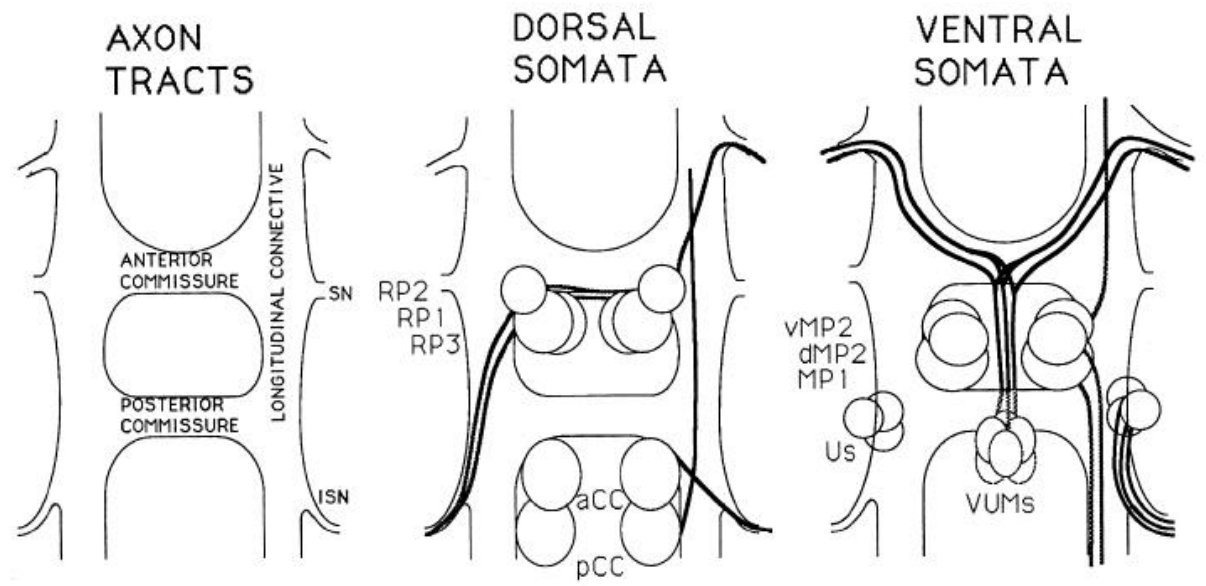

Figure 1. Cell body locations of embryonic neurons described in this paper which pioneer axon tracts in the developing Drosophila CNS. The major axon tracts, identified at left, are shown relative to the positions of 5 groups of neuronal cell bodies. Middle, Neurons near the dorsal surface of the CNS, the level where the axon tracts form. Right, Cell bodies found ventral to the major axon tracts. See text for details. of neurons (Campos-Ortega and Hartenstein, 1985). The axons that pioneer the first tracts of the Drosophila CNS extend from medial and (with some exceptions) dorsal neurons born early during CNS neurogenesis (i.e., before $9 \mathrm{hr}$ of development).

The cell body locations of 5 major groups of pioneer neurons are represented schematically in Figure 1. The RP neurons are a bilaterally represented trio of dorsal neurons between the anterior and posterior commissures. The neurons RP1 and RP3 pioneer 2 separate commissural fascicles that join together as they turn posteriorly and then laterally to join the contralateral intersegmental nerve (ISN). RP2 pioneers a fascicle that extends anterior and laterally to the ipsilateral ISN of the next anterior segment. The pair of sibling aCC and pCC cells are located immediately posterior to the posterior commissure. The anterior corner cell $(\mathrm{aCC})$ pioneers the ISN and the posterior corner cell $(\mathrm{pCC})$ is one of the pioneers of the longitudinal connective. These cells were first identified and named in Drosophila by their homology to identified grasshopper neurons (Thomas et al., 1984).

Slightly ventral and posterior to the RP neurons are 3 neurons that are the progeny of midline precursors 1 and 2 (MP1 and MP2): the MP1, dMP2, and vMP2 neurons. The MP1 and dMP2 axons extend posteriorly and the vMP2 axon extends anteriorly as all 3 help pioneer the longitudinal axon tracts. The most ventral of the pioneer neurons described in this paper are 6 ventral unpaired median (VUM) neurons located beneath the posterior commissure; their axons pioneer a median fascicle before they bifurcate and unite with the RP2 fascicles on each side as they head anteriorly and laterally towards the ISN. Finally, the Us (U1-U3), located lateral and ventral to the MPs, produce axons which follow aCC in the formation of the ISN. All of these neurons are homologous to pioneers of the same tracts in the grasshopper embryo. We have studied the axon extension and fasciculation choices of these pioneer neurons in Drosophila using serial electron microscopy, with the aim of determining whether these 2 insects employ the same cell-cell recognition events in the establishment of their axon tracts.

In Drosophila, the pioneer neurons begin axonogenesis between 9:45 and 10:00 hr of development, and their axons extend beyond their segment within $30 \mathrm{~min}$. Thus, the axon scaffold of the embryo is constructed over a period of less than an hour. While the growth cones of pioneer neurons are navigating through the developing neuropil, the cell bodies they make contact with are also in motion, because the period of tract formation co- incides with the late phase of germ band shortening. The pioneers emerge when each segment is $36 \mu \mathrm{m}$ long, but the segment is $15 \%$ shorter $1 \mathrm{hr}$ later, and is more than $30 \%$ shorter at hatching.

The developing neuropil of each hemisegment is bordered by dorsal glial cells and early differentiating neurons. During the period under study, the breadth of the neuropil is $4-10 \mu \mathrm{m}$ and is almost entirely occupied by axons, growth cones, and filopodia. During this period, every cell surface bordering the neuropil of each segment is within filopodial grasp of every growth cone in that segment.

Below we examine the trajectory and fasciculation choices of these 5 groups of neurons as they pioneer the major tracts and in so doing construct the axon scaffold of the Drosophila CNS.

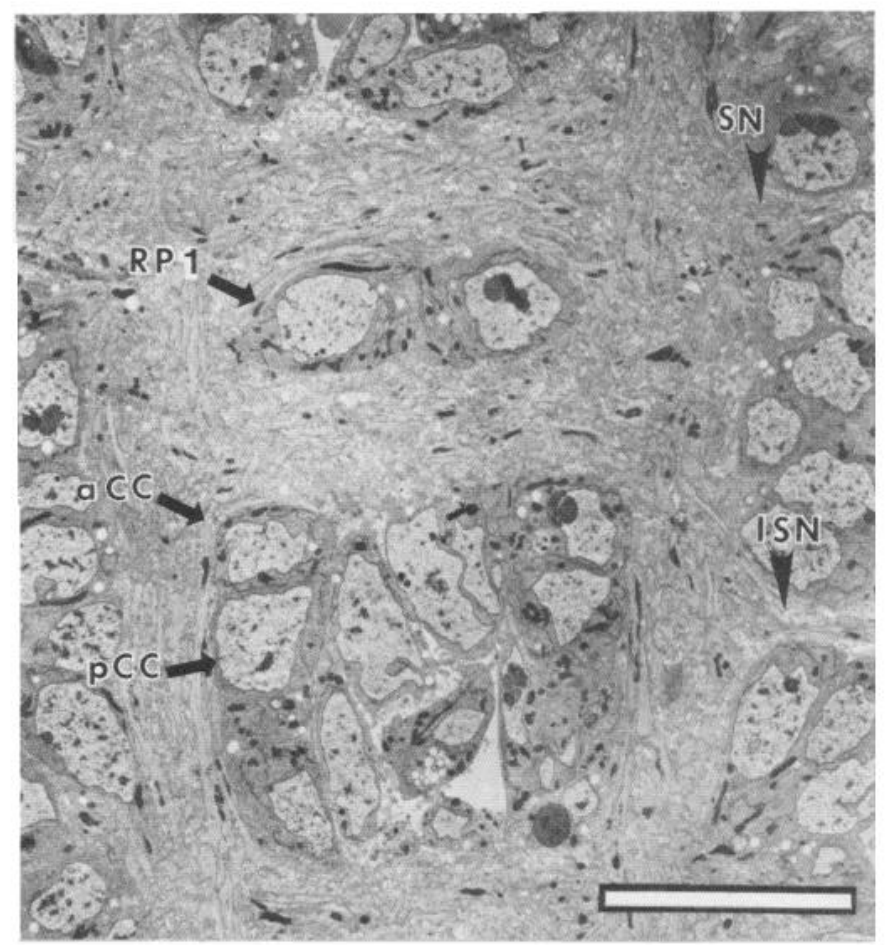

Figure 2. Frontal section near the dorsal surface of a single segment at $11 \mathrm{hr}$, showing the major axon tracts and the cell bodies of 3 of the dorsal pioneer neurons described in this paper: RP1, aCC, and pCC. Anterior is at top. Scale bar, $10 \mu \mathrm{m}$. 

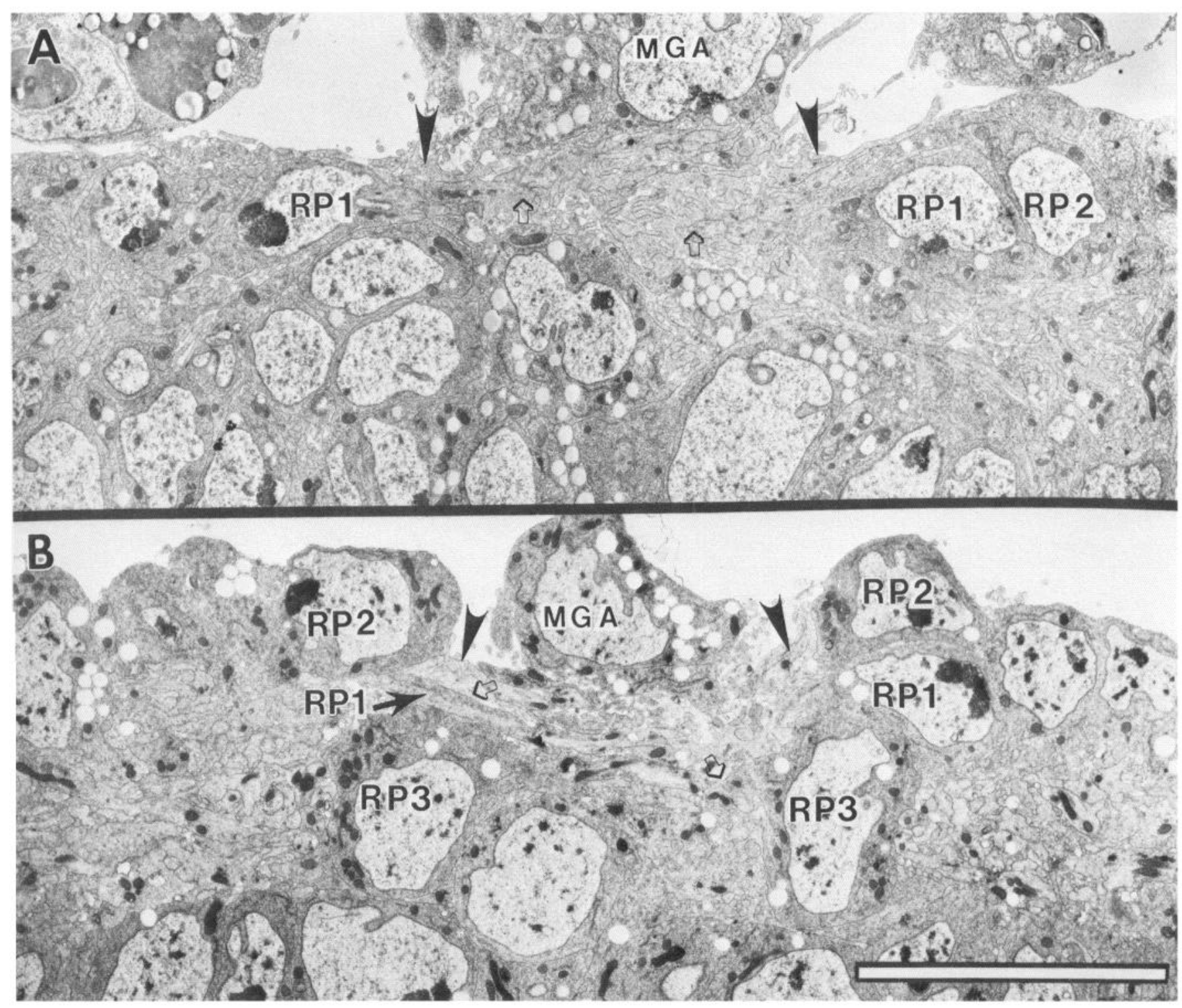

Figure 3. Medial migration of the cell bodies of the RP neurons along the dorsal surface toward the midline. In cross section through the midline $(A)$ at 9:45 hr of development, the RP1 and RP2 neurons are still located laterally, over the developing longitudinal tract. The RPs are extending processes medially through the nascent anterior commissure. Serial reconstruction of this segment shows that the leading processes of both RP1 neurons are in contact with each other. Note that the longitudinal tract and anterior commissure contain growth cones and filopodia only at this early stage. Thirty minutes later in development $(B)$, all 3 RP cells are considerably closer to the midline, and have prominent axons that contact their contralateral homologs. RP2 has moved dorsally and projects its axon anteriorly. Note that the anterior commissure and longitudinal connectives contain numerous axons. Solid arrowheads in both $A$ and $B$ show growth cone processes of the VUM neurons contacting the RP2 cell bodies. Open arrows indicate RP cell processes in $A$ and contacts between RPs and contralateral RP axons in $B$. Scale bar, $10 \mu \mathrm{m}$.

\section{$R P$ neurons and the formation of the anterior commissure}

The RPs are a trio of neurons (RP1-RP3) with prominent dorsal cell bodies. Two of the RP cell bodies (RP1 and RP3) are located between where the anterior and posterior commissures form; RP1 lies dorsal to and directly over RP3. The anterior surface of the RP1 and RP3 cell bodies are bounded by tight fascicles of axons that are among the first in the anterior commissure; these fascicles are pioneered by the growth cones of the contralateral RPs. After crossing the midline, these axons turn posteriorly along the longitudinal tract towards the ISN (Fig. 2). These 2 fascicles (the RP1 fascicle and the RP3 fascicle) are some of the first pioneered through the anterior commissure.
Other fascicles are pioneered by as yet unidentified neurons located lateral to the anterior commissure.

Although the cell bodies of the RP neurons are ultimately located quite medially, they are born lateral to the future location of the longitudinal connectives. Before axonogenesis, a $5-\mu \mathrm{m}$-long amoeboid process precedes each cell as the cell bodies migrate medially, passing between the anterior pair of longitudinal tract glia (LG1 and LG2; see companion paper) on the way. When the RPs reach the posterior lateral corner of the nascent anterior commissure, the leading processes of RP1 and RP3 elongate medially, to contact the cytoplasmic extensions of their contralateral homologs as shown in Figure $3 \mathrm{~A}$. The leading process of each RP cell thins out to axon caliber $(<0.3$ 


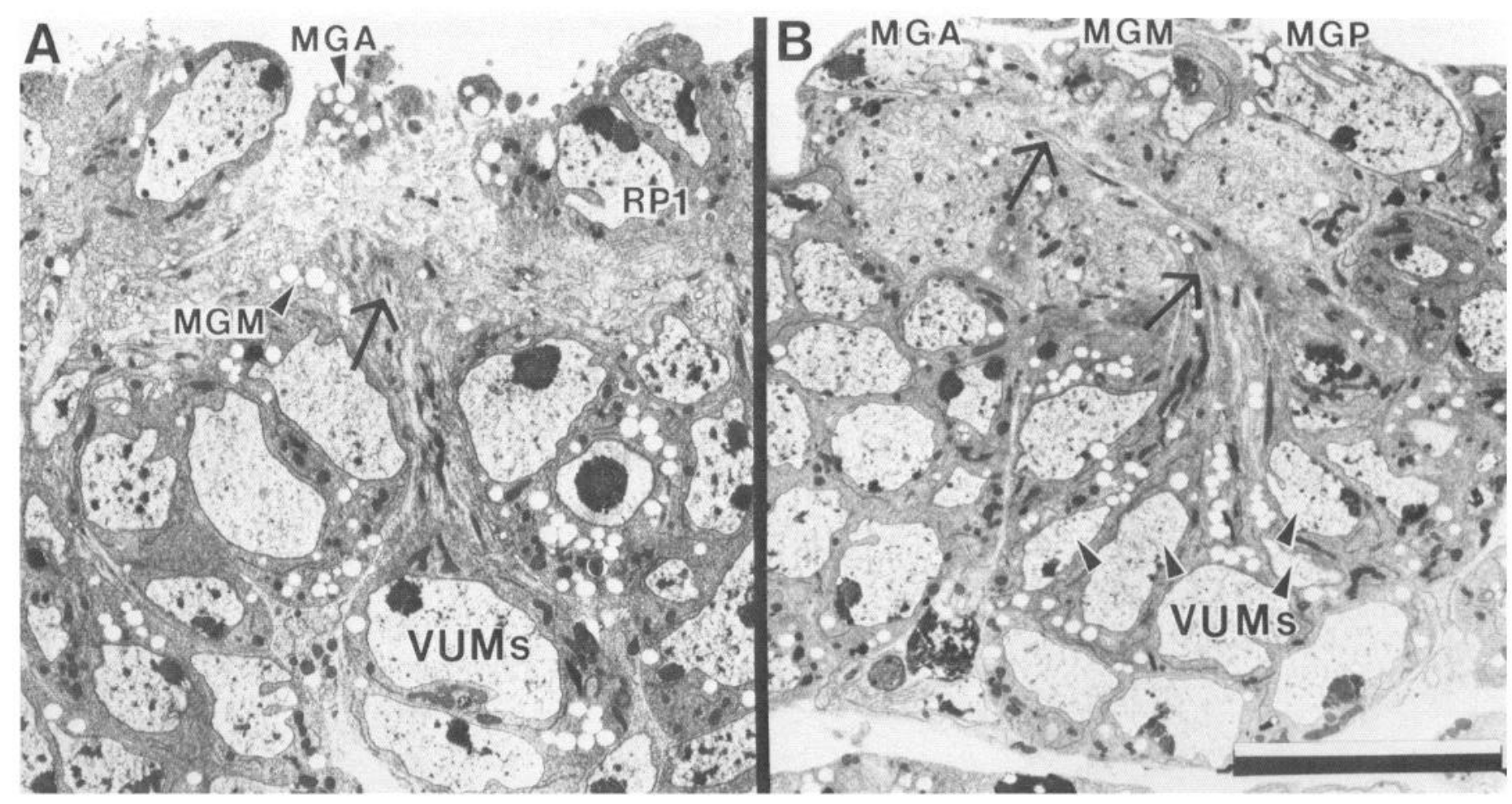

Figure 4. Projection of the VUM neurons. $A$, Tangential cross section (dorsal is more anterior than ventral) soon after $10 \mathrm{hr}$ catches the axon bundle of the VUMs (arrow) as they project dorsally and anteriorly into the anterior commissure. The VUM bundle is bordered by the MGM cell at the midline and contacts the ventral surface of MGA, awaiting contact with the medially migrating RP2 soma. $B$, In sagittal view, after $11 \mathrm{hr}$, the VUM axon bundle projects between the MGM glia to contact the MGA cell dorsally, before bifurcating and projecting along the axons of both RP2 neurons. Scale bar, $10 \mu \mathrm{m}$.

$\mu \mathrm{m}$ diameter), as the growth cone tips of the axons of RP1 and RP3 extend around the cell bodies of their contralateral homologs. This behavior also acts to pull all $3 \mathrm{RP}$ cell bodies medially toward each other, compressing extensions of the midline glia (MGM) between them (Fig. 3B). The growth cones of RP1 and RP3 turn posteriorly around their homologs and pioneer a fascicle that heads towards the contralateral ISN. The RP fascicle extends posteriorly, adhering to the ventral surface of the longitudinal tract glia, until contacting the SBC before turning laterally as part of the ISN.

The leading process of RP2 does not continue medially during the final medial migration of the RP1 and RP3 cell bodies. Rather, during the later stages of RP migration, the leading process of RP2 develops into an axon, whose growth cone extends anteriorly towards the ipsilateral ISN of the next anterior segment.

\section{Median fascicle of the VUM neurons}

The VUMs are 6 prominent neurons at the ventral midline that are likely to be the progeny of midline precursors homologous to MP4-MP6 in the grasshopper (Bate and Grunewald, 1981). The VUM growth cones pioneer an ascending fascicle that bifurcates as it enters the anterior commissure. Early in development (before $11 \mathrm{hr}$ ), the VUM cell bodies are ventral to the posterior commissure. However, the nerve tracts continue to shorten relative to the cell bodies of CNS neurons, so that after $12 \mathrm{hr}$, the VUM cell bodies are ventral and posterior to the posterior commissure. Before the RP neurons have completed their medial migration, the growth cones of the VUMs have already extended vertically to contact the MGM cells dorsal and anterior to them. In reciprocation, the MGM cells project cytoplasmic extensions ventrally to enclose the VUM fascicle (Fig. 4). Once the growth cones of the VUMs reach the anterior commissure, they contact the anterior midline glia (MGA) and cease extension. Extension resumes 20 min later when the growth cones contact the cell bodies of the medially migrating RP2 neurons (Fig. 3, $A, B$ ). The growth cones of the VUM neurons bifurcate as they contact both RP2 cells and thus generate a bilateral projection. From here on, each VUM growth cone fasciculates with its respective RP2 axon as it extends towards the ISN.

\section{Pioneers of the longitudinal connective}

The tracts of the longitudinal connective are pioneered by 2 ascending growth cones and 2 descending growth cones, which meet near the posterior end of the anterior of each pair of adjacent segments. Each projection is considered separately below.

\section{Anterior projection pioneering the longitudinal connective}

The pCC neuron is located medial to the longitudinal connective, and posterior to the posterior commissure. Its axon projects anteriorly through the neuropil of its own segment to contact a lateral extension of the vMP2 cell at 9:45 hr of development (Fig. $5 A$ ). The growth cones of vMP2 and pCC proceed anteriorly to make the initial contact with the next anterior segment, on the surface of glial cell LG5 (Fig. $5 B$ and companion paper). The pCC is followed at 10:15 hr by the first of 3 follower cells (FP) which fasciculate with the pCC axon and follow the same trajectory. The FP cell bodies are located ventral and anterior 


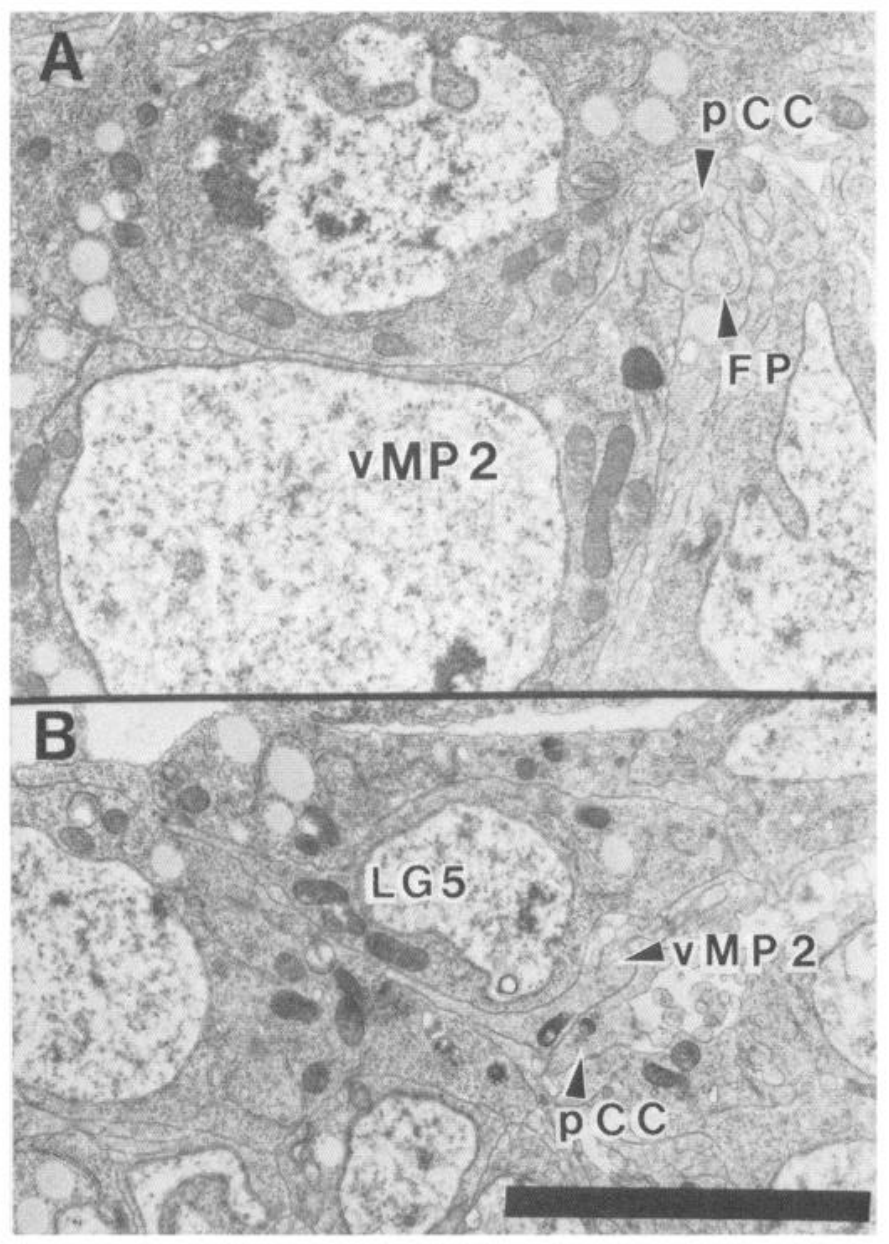

Figure 5. Interaction of pCC and vMP2 growth cones. $A$, The axon of pCC and its companion, FP, contacts the vMP2 cell body (in cross section) at the point of vMP2's initial axonal segment. $B$, The growth cones of vMP2 and pCC extend anteriorly in tandem along the surface of glial cell LG5. Both micrographs are from the same segment, in an embryo at 10:15 hr of development. Medial is at left for Figures 5-7 and 10 . Scale bar, $4 \mu \mathrm{m}$.

to pCC and make somal contact with midline precursor 1 (MP1; Fig. 6, $A, B)$.

\section{Posterior projection pioneering the longitudinal connective}

Immediately posterior to vMP2 is its sibling cell, dMP2, and MP1. The axons from these 2 cells fasciculate together and pioneer the first posteriorly extending longitudinal tract. At 9:50 hr, MP1 (and within $10 \mathrm{~min}, \mathrm{dMP} 2$ ) extends dorsally and laterally towards the neuropil to contact the axons of pCC and FP (Fig. 6A). The growth cones of MP1 and dMP2 then extend posteriorly along the axon of pCC until they contact the cell bodies of aCC and pCC. In Figure $6 B$, a tangential section visualizes the contacts of the MP1 and dMP2 axons with both the $\mathrm{pCC}$ axon and the aCC cell body. The MP1/dMP2 growth cones do not show any selectivity between the surfaces of the cell bodies of the aCC and pCC neurons and are generally found in contact with both (Fig. 6, C,D). However, posterior to the aCC cell body, the MP1/dMP2 growth cones show no affinity for the aCC axon (which pioneers the ISN) and rather contact the pCC cell body exclusively.

The growth cones of the MP1/dMP2 neurons reach the pos- terior edge of the pCC cell body shortly after $10 \mathrm{hr}$, where they stay until at least 10:15 hr. In serial reconstructions over this period, the MP1/dMP2 growth cones are found in contact with pCC, while other axons extend the equivalent of one-half segment. At this time, the anteriorly extending growth cones of vMP2 and pCC from the next posterior segment reach the level of the pCC cell body, where they contact the pCC cell body and the MP1/dMP2 growth cones. Figure $7 A$ shows the MP1/dMP2 growth cones immediately before and Figure $7 B$ immediately after the arrival of the vMP $2 / \mathrm{pCC}$ growth cones. At this point, all of the pioneers of the longitudinal tract are in contact with each other. We have not established whether the posteriorly extending MP1/dMP2 growth cones continue to fasciculate with the vMP2/pCC axon fascicle as they continue through the next posterior segment. The morphology of the longitudinal tract pioneers at 10:15 hr of development is summarized in a serial section reconstruction of the pioneer neurons in Figure 8.

\section{Pioneers of the ISN}

At 9:45 hr of development, the aCC cell body is less than $5 \mu \mathrm{m}$ anterior to the origin of the ISN. The growth cone of aCC extends posteriorly and laterally through the dorsal neuropil to contact the segment boundary cell (SBC), a glial cell that later ensheaths the ISN, and continues to the periphery. Germ band shortening brings the aCC closer to the SBC after formation of the IS nerve (Fig. 6D). Before $10 \mathrm{hr}$ of development, another 3 axons, the Us (U1-U3), join the ISN. The Us are a group of neurons located directly under the neuropil immediately anterior to aCC. They project their first growth cones dorsally to contact the dorsal glia and the cell body and axon of aCC at 9:45 hr. The U axons maintain their contact with aCC's cell body throughout this period of development (Fig. 7). The growth cones of the Us follow aCC to the SBC and continue to the PNS (Fig. 6D). Once the Us contact the SBC, they may cease to contact the aCC but always maintain contact with the SBC. The morphology of the ISN pioneers from a serial section reconstruction at $10: 15 \mathrm{hr}$ is shown in Figure 9.

\section{Discussion}

The first axon pathways in the Drosophila CNS are established by a small number of neurons between 9:45 and 10:15 hr of development. In this paper we have examined the neurons that pioneer 3 of these CNS pathways: the longitudinal tract, the ISN, and the anterior commissure. These neurons appear homologous to the neurons that establish the same pathways in the grasshopper (Thomas et al., 1984). In many respects, the trajectories and fasciculation choices of Drosophila neurons are nearly identical to those in the grasshopper, but there are a few important differences that provide insights into the mechanisms of pathway choices and growth cone guidance.

An interesting timing difference is revealed during the formation of the ISN. Figure 10 shows that different neurons pioneer the ISN in the 2 insect species, although there is no change in specific surface affinities. In the grasshopper, the first 2 Us (U1 and U2) extend posteriorly through the neuropil until they contact the SBC. The Us then turn laterally on the SBC surface to pioneer the ISN (Bastiani and Goodman, 1986). A third U growth cone extends along this fascicle shortly thereafter. In the grasshopper, the aCC growth cone must make contact with and fasciculate with the $U$ axons in order to turn posteriorly and find the SBC. If the Us are ablated, the aCC fails to find the SBC and instead extends anteriorly (du Lac et al., 1986). In 


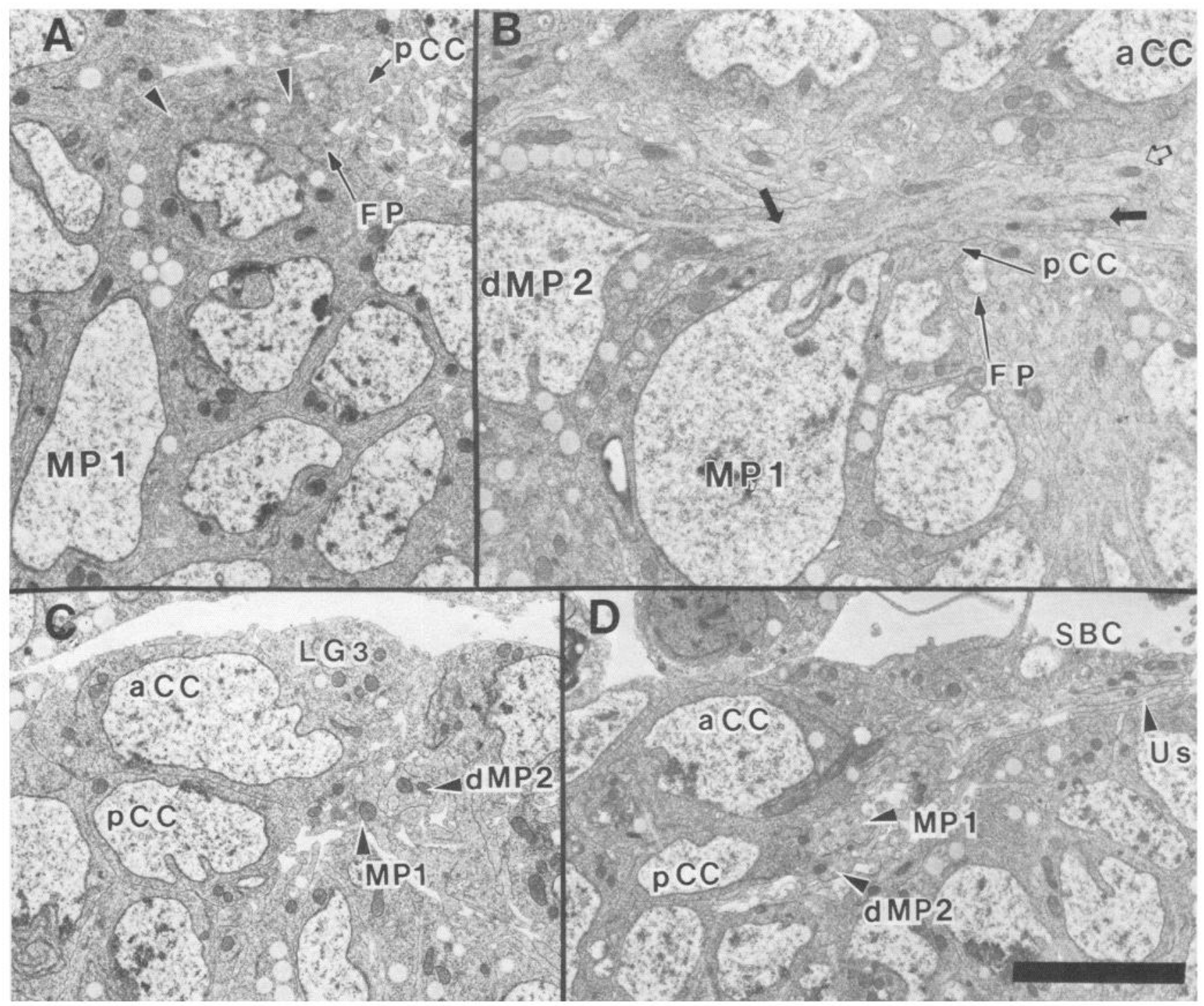

Figure 6. Interactions of the MP1 and dMP2 growth cones with the aCC and pCC neurons. $A$, The medially located MP1 extends its axon dorsally and laterally (arrowheads) to contact the axons of pCC and FP at 9:50 hr of development. B, A tangential section at a comparable location at 10:15 hr shows the MP1 (open arrow), dMP2 (solid arrows) axons in contact with the pCC axon and the aCC cell body. $C$, At $7 \mu \mathrm{m}$ posterior to micrograph $(A)$ at 9:50 hr, the growth cones of MP1 and dMP2 are in contact with the cell body of aCC. $D$, Posterior to micrograph $(B)$ at 10:15 hr, the growth cones of MP1 and dMP2 are in contact with the cell bodies of both aCC and pCC. The Us are fasciculated along the surface of the SBC. Scale bar, $A-C, 4 \mu \mathrm{m} ; D, 5.5 \mu \mathrm{m}$.

Drosophila, aCC makes contact with the SBC before the Us have extended posteriorly, and pioneers the ISN. The Us maintain an affinity for the surface of aCC, and follow this cell posteriorly to the SBC. The affinity that aCC and the Us have for each other is preserved, but timing differences exploit this affinity in a different way in each species. In the grasshopper, this contact is essential for aCC to take its correct trajectory; in Drosophila it is not; rather, it is the Us that follow the aCC.

One major species-specific difference between grasshopper and Drosophila is in the affinity of the longitudinal tract pioneers vMP2 and pCC (Fig. 10). In grasshopper, the MP1/dMP2 fascicle (which contains the pCC axon) and the vMP2 fascicle are 2 distinct longitudinal pathways, whereas in Drosophila these same 4 neurons pioneer a single combined longitudinal pathway. This difference not only reduces the number of initial longitudinal fascicles in Drosophila relative to grasshopper, but it also changes the relative timing and sequence of cell recognition events during longitudinal tract formation. In both species, the vMP2 growth cone extends into the next anterior segment along the surface of a dorsal glial cell from the next segment (Bastiani et al., 1986; Jacobs and Goodman, 1989). However, in Drosophila, but not grasshopper, pCC extends anteriorly in tandem with the vMP2 to pioneer the first longitudinal tract. In contrast, in the grasshopper, the pCC does not extend anteriorly along the vMP2, but rather it waits in the middle of the segment until the MP1/dMP2 growth cones from the next anterior segment come within reach and then extends anteriorly along them. Thus, in grasshopper, the first intersegmental contact is made by the descending MP1/dMP2 axons, whereas in Drosophila it is made by the ascending vMP2/pCC axons. Whereas the $\mathrm{pCC}$ waits for the descending MP1/dMP2 growth cones from the next anterior segment in the grasshopper, in the fly the MP1/dMP2 growth 


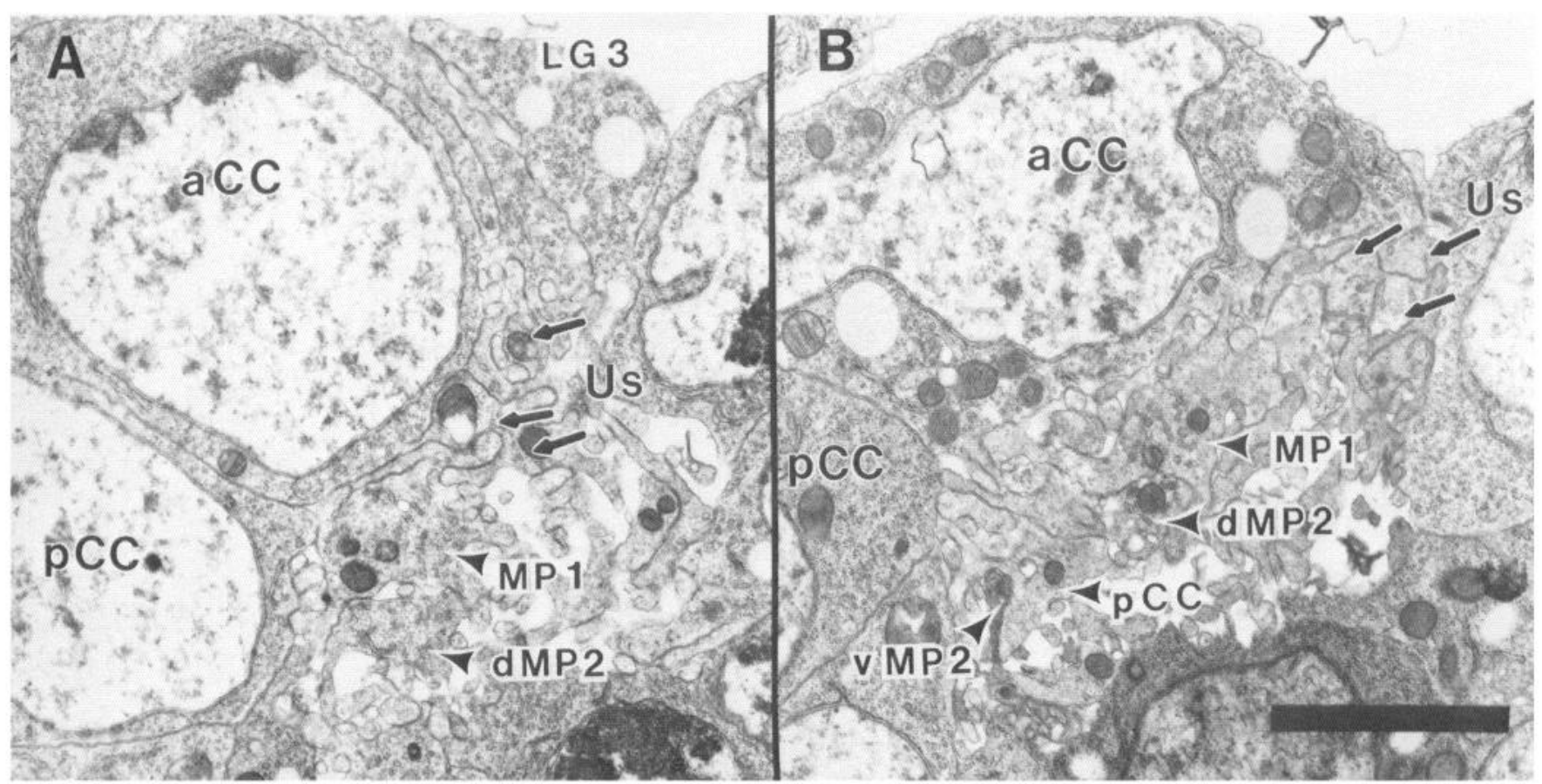

Figure 7. Meeting of the anterior and posterior extensions of the longitudinal tract. $A$, In a $10 \mathrm{hr}$ embryo, the growth cones of MP1 and dMP2 are in contact with both aCC and pCC, while the Us are in contact with aCC only. $B$, In the same embryo as $A$, but 2 segments anterior, the ascending growth cones of pCC and vMP2 make contact with both the descending growth cones of MP1 and dMP2 from the next segment, as well as the soma of pCC of the next segment. Scale bar, $2 \mu \mathrm{m}$.

cones wait at the level of the pCC cell body for the ascending vMP2/pCC growth cones from the next posterior segment.

The differences in longitudinal tract formation between these 2 insect species can be accounted for by differences in cell affinities. In Drosophila, the pCC growth cone displays a high affinity for the vMP2 axon, whereas in grasshopper it does not. In Drosophila, the MP1/dMP2 growth cones wait for and fasciculate with the vMP2/pCC axons, whereas in grasshopper they form 2 distinct longitudinal axon fascicles. Some of these differences may reflect actual evolutionary changes in the surface molecules expressed by individual growth cones, whereas other differences may simply reflect changes in the physical environment in which otherwise identical neurons differentiate.

In considering these issues, it is important to consider the dramatic structural differences between the developing neuropil in the grasshopper and Drosophila. In grasshopper, the MP1/ dMP2 growth cones explore a channel about $20 \mu \mathrm{m}$ wide and $20 \mu \mathrm{m}$ deep, whereas in Drosophila the dimensions are less than one-tenth that size (Fig. 11); in grasshopper, the distance from segment border to segment border is over $300 \mu \mathrm{m}$, whereas in Drosophila it is about one-tenth that length.

One consequence of the small neuropil size in Drosophila is the forced proximity of all axon fascicles. This difference is demonstrated in Figure 11 in a comparison of the developing longitudinal pathways at the level of the aCC cell body at equivalent developmental stages in the grasshopper (top) and Drosophila (bottom). In the grasshopper, 3 major axon tracts (the U, MP1/dMP2, and vMP2 fascicles) are separated by several microns of open space. In contrast, in the fly, the same fascicles are separated by fractions of a micron, often coming into physical contact with each other over much of their length.

A second consequence of the relative size difference is the dramatic difference in the sampling environment of an individual filopodium in each species. The grasshopper has a very open neuropil, permitting growth cones and filopodia to explore the extracellular environment in many directions. The dorsal surface of the neuropil, for example, provides unlimited space for filopodial contact with the basement membrane and membranous projections from laterally placed glia (Fig. 11, top). Drosophila growth cones and their filopodia are confined to a narrow channel, permitting exploration and extension only along the length of the axon tracts. The dorsal surface of the neuropil is comparatively small and dominated by glial cells, whose ventral surface is saturated with filopodial and axon contacts (Fig. 11, bottom and companion paper).

However, at this early stage of pathway formation, the typical neuron diameter in Drosophila is about $2-4 \mu \mathrm{m}$, and the length of each segment about $30 \mu \mathrm{m}$. A typical Drosophila filopodium is about $15 \mu \mathrm{m}$ in length and can thus sample the environment for about $4-8$ cell diameters, or about $50 \%$ the length of a segment. In contrast, in grasshopper, neuronal cell bodies are about $15 \mu \mathrm{m}$ in diameter, and the length of each segment is about $300 \mu \mathrm{m}$. Grasshopper filopodia are typically $30 \mu \mathrm{m}$ in length (Taghert et al., 1982). Although a grasshopper growth cone via its filopodia can sample a volume 8 times larger than that of a typical Drosophila growth cone, it can only sample about $10 \%$, as compared with $50 \%$ in Drosophila, the length of a segment in any direction.

Thus, pioneering growth cones in Drosophila can contact distant glial cells and axon fascicles with ease in their own or neighboring segments that are beyond the reach of homologous grasshopper growth cones. This is certainly true in the pioneering of the ISN, where in grasshopper the SBC is beyond the reach of the aCC growth cone. In grasshopper, the aCC waits 


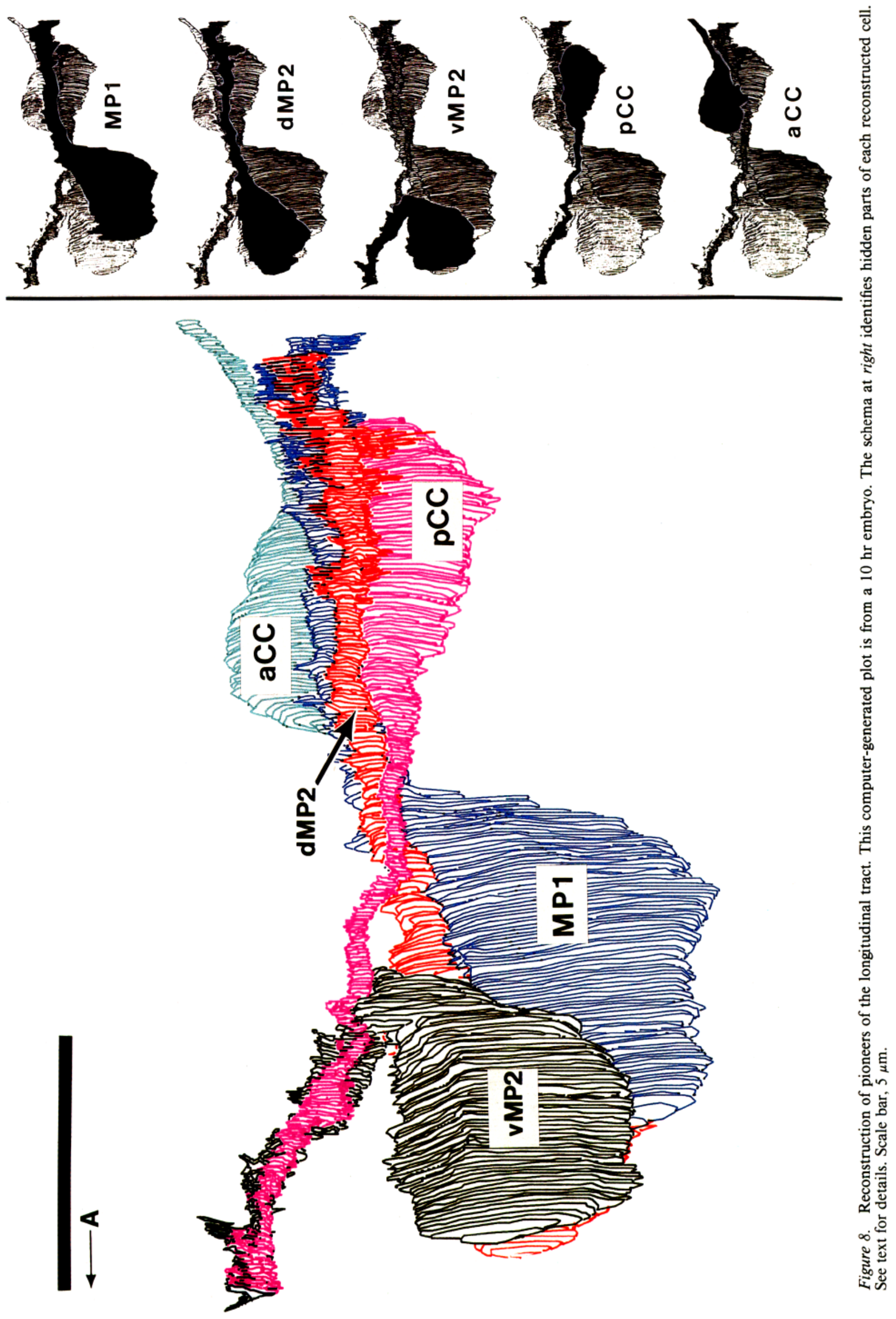


Figure 9. Reconstruction of pioneers of the ISN. From the same series as used in Figure 8. Scale bar, $2 \mu \mathrm{m}$.

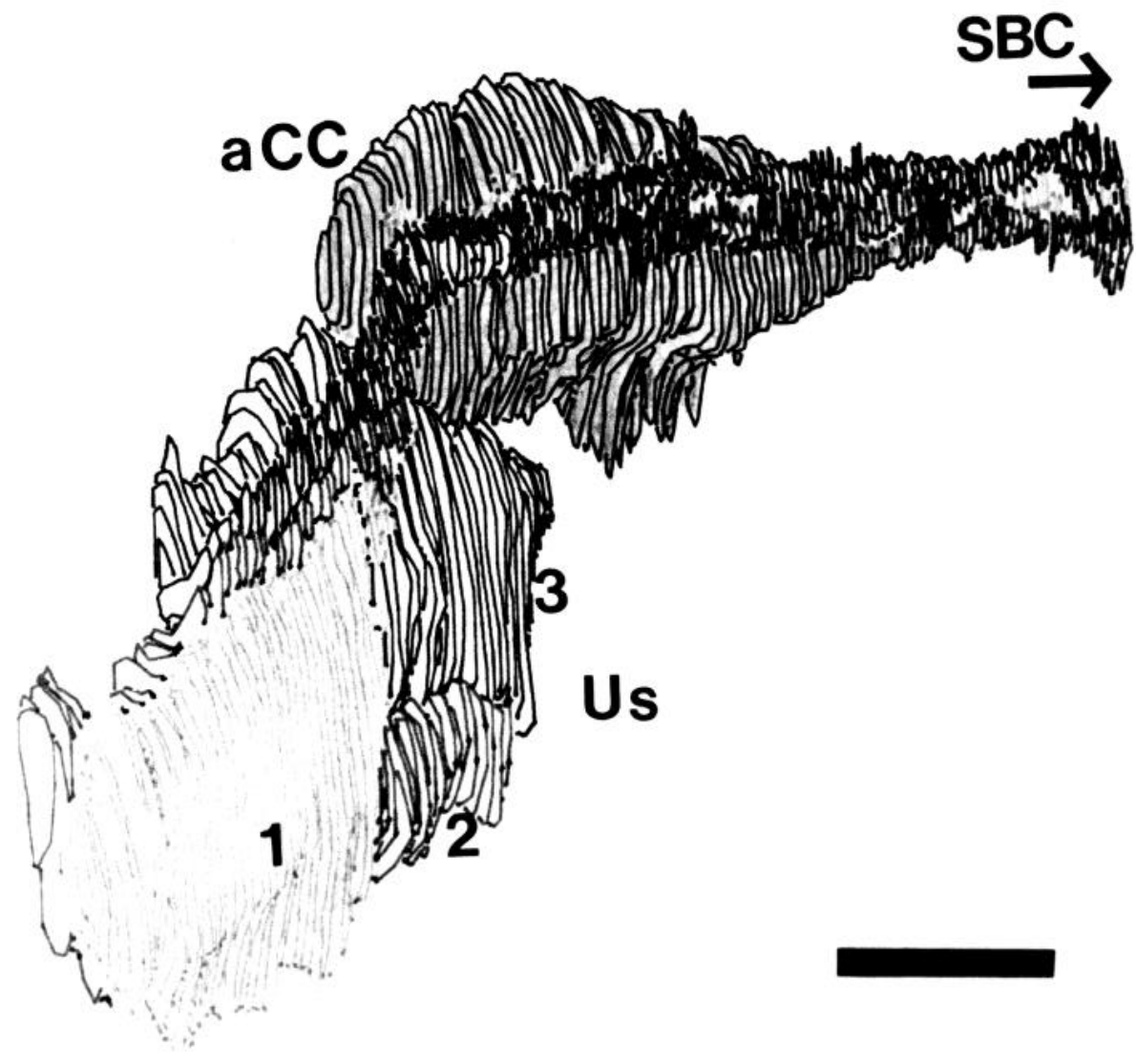

for the $\mathrm{U}$ axons, whereas in Drosophila, the aCC growth cone without delay extends along the surface of the SBC, which is only a few microns away. One consequence of these short distances in Drosophila as compared with grasshopper is that certain transient contacts and affinities may not be as important in the fly where growth cones can reach relatively longer distances to find their next pathways and targets. For example, in grasshopper, ablation of the Us leads to abnormal behavior of the aCC growth cone because it is too far away to contact the $\mathrm{SBC}$ on its own; we would predict that such an ablation in the fly would have no effect on the aCC and little effect if any on subsequent growth cones.

In retrospect, it is fortunate that the first laser ablation experiments were performed in grasshopper, where the results consistently revealed specific cellular affinities because growth cones were typically far away from their next pathway choice.
Figure 10. Schematic of the first axon fascicles in the grasshopper and Drosophila CNS at $37 \%$ or $10 \mathrm{hr}$ of development, respectively. Note that vMP2 pioneers its own tract in the grasshopper, while in Drosophila it fasciculates with pCC. In the grasshopper, the first 2 Us pioneer the ISN. They are joined later by aCC and another U. In contrast, in Drosophila, aCC pioneers the ISN, and the first 3 Us arrive together afterwards.
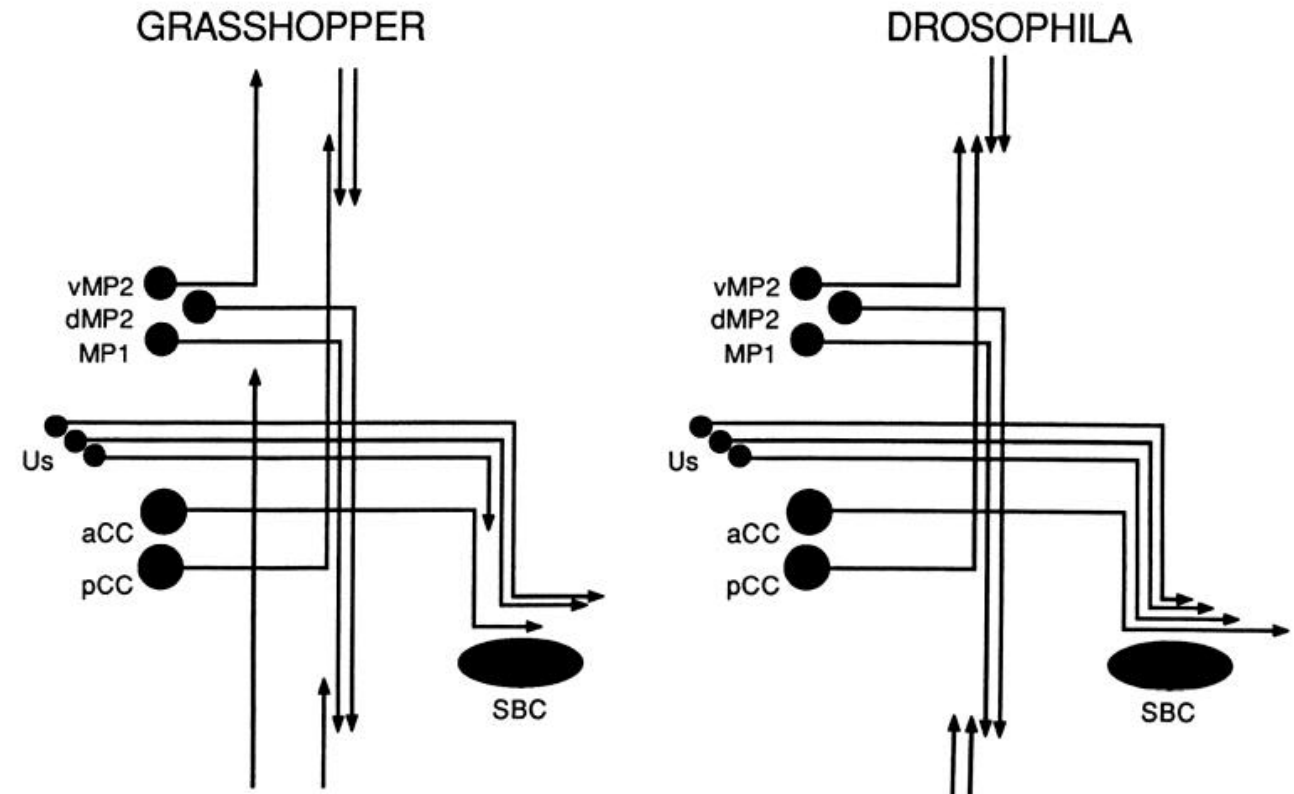


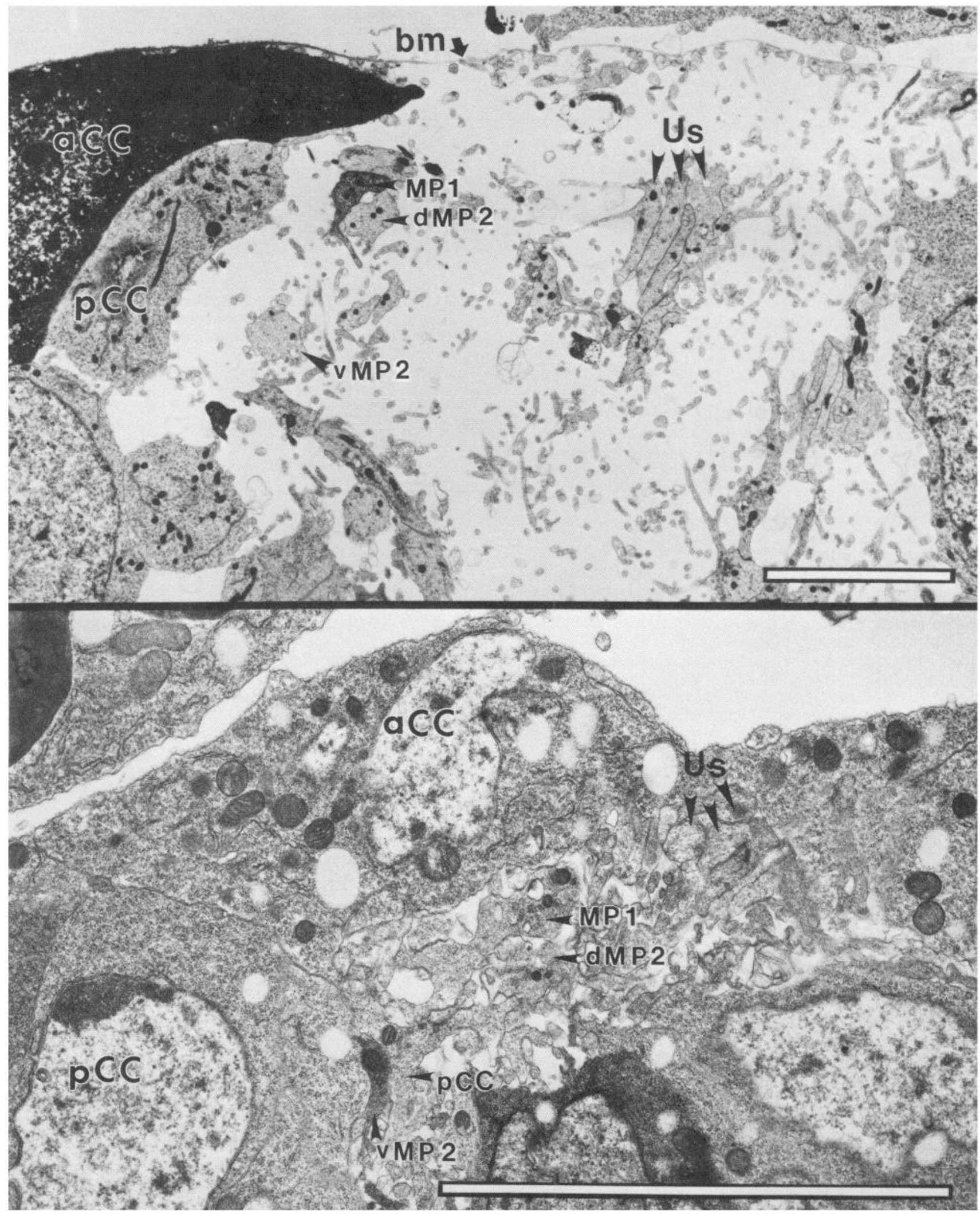

Figure 11. The longitudinal tract pioneers of grasshopper and Drosophila at equivalent times and locations. Top, A cross section through the grasshopper neuropil at the level of aCC and pCC at 37\% of development shows the MP1/dMP2, vMP2 and U fascicles widely separated in a relatively open space. Numerous filopodia occupy the space or contact the dorsal basement membrane $(\mathrm{bm})$. The aCC cell body has been injected with HRP and reacted with diaminobenzidine. Bottom, A cross section through an equivalent location in the Drosophila neuropil showing the same 3 fascicles in the same relative positions as found in grasshopper. Note, however, that aCC is in physical contact with the U fascicle and the MP1/dMP2 fascicle, and the vMP2/pCC fascicle is in contact with both the MP1/dMP2 fascicle and the pCC cell body. Scale bars, $4 \mu \mathrm{m}$. 
Such ablations in Drosophila, whether done by laser beam or genetic perturbation, might lead to more subtle phenotypes because of the proximity of growth cones to their next pathway choices. Thus, generating such strong phenotypes in Drosophila as were produced by cell ablations in grasshopper might require removing multiple cellular pathways or multiple molecular pathway markers.

In summary, we have found differences in cell-cell affinity, in the timing, and in the polarity of cell-cell contacts by comparing axon fasciculation choices of the pioneers of the major CNS tracts in Drosophila and grasshopper. Although there are some differences, it is nevertheless quite fascinating that these homologous neurons, after 300 million years of evolutionary divergence, still differentiate in very much the same manner, including identical axon trajectories. Homologs to the pioneer neurons examined here have been found in all arthropod species examined (Thomas et al., 1984). A likely explanation for this striking conservation of early neuronal development is that it relies upon strongly conserved cell-recognition mechanisms, robust enough to function through a broad range of rates of embryonic development and sizes of embryonic nervous systems.

\section{References}

Bastiani, M. J.; and C. S. Goodman (1986) Guidance of neuronal growth cones in the grasshopper embryo. III. Recognition of specific glial pathways. J. Neurosci. 6: 3542-3551.

Bastiani, M. J., J. A. Raper, and C. S. Goodman (1984) Pathfinding by neuronal growth cones in grasshopper embryos. III. Selective affinity of the $G$ growth cone for the $P$ cells within the A/P fascicle. $J$. Neurosci. 4: 2311-2328.

Bastiani, M. J., C. Q. Doe, S. L. Helfand, and C. S. Goodman (1985) Neuronal specificity and growth cone guidance in grasshopper and Drosophila embryos. TINS 8: 257-266.

Bastiani, M. J., S. du Lac, and C. S. Goodman (1986) Guidance of neuronal growth cones in the grasshopper embryo. I. Recognition of a specific axonal pathway by the pCC neuron. J. Neurosci. 6: 3518 3531 .

Bate, C. M., and E. B. Grunewald (1981) Embryogenesis of an insect nervous system. I. A map of the thoracic and abdominal neuroblasts in Locusta migratoria. J. Embryol. Exp. Morphol. 35: 107-123.
Blair, S. S., and J. Palka (1985) Axon guidance in the wing of Drosophila. Trends Neurosci. 8: 284-288.

Blair, S. S., M. A. Murray, and J. Palka (1987) The guidance of axons from transplanted neurons through aneural Drosophila wings. J. Neurosci. 7: 4165-4175.

Bodmer, R., and Y.-N. Jan (1987) Morphological differentiation of the embryonic peripheral neurons in Drosophila. Roux's Arch. Dev. Biol. 196: 69-77.

Campos-Ortega, J. A., and V. Hartenstein (1985) The Embryonic Development of Drosophila melanogaster. Springer-Verlag, Berlin.

Caudy, M., and D. Bentley (1986a) Pioneer growth cone morphologies reveal proximal increases in substrate affinity within leg segments of grasshopper embryos. J. Neurosci. 6: 364-379.

Caudy, M., and D. Bentley (1986b) Pioneer growth cone steering along a series of neuronal and non-neuronal cues of different affinities. J. Neurosci. 6: 1781-1795.

du Lac, S., M. J. Bastiani, and C. S. Goodman (1986) Guidance of neuronal growth cones in the grasshopper embryo. II. Recognition of a specific axonal pathway by the aCC neuron. J. Neurosci. 6: 35323541.

Ghysen, A., C. Dambly-Cjaudiere, E. Aceves, L.-Y. Jan, and Y.-N. Jan (1986) Sensory neurons and peripheral pathways in Drosophila embryos. Roux's Archiv. Dev. Biol. 195: 281-289.

Jacobs, J. R., and C. S. Goodman (1989) Embryonic development of axon pathways in the Drosophila CNS: I. A glial scaffold appears before the first growth cones. J. Neurosci. 9: 2402-2411.

Jan, Y. N., A. Ghysen, I. Christoph, S. Barbel, and L. Y. Jan (1985) Formation of neuronal pathways in the imaginal discs of Drosophila melanogaster. J. Neurosci. 5: 2453-2464.

Kuwada, J. Y. (1986) Cell recognition by neuronal growth cones in a simple vertebrate embryo. Science 233: 740-746.

Steller, H., K.-F. Fischbach, and G. M. Rubin (1987) Disconnected: A gene required for neuronal pathways formation in the visual system of Drosophila. Cell 50: 1139-1153.

Taghert, P. H., M. J. Bastiani, R. K. Ho, and C. S. Goodman (1982) Guidance of pioneer growth cones: Filopodial contacts and coupling revealed with an antibody to Lucifer yellow. Dev. Biol. 94: 391-399.

Thomas, J. B., M. J. Bastiani, M. Bate, and C. S. Goodman (1984) From grasshopper to Drosophila: A common plan for neuronal development. Nature 310: 203-207.

Walthall, W. W., and M. Chalfie (1988) Cell-cell interactions in the guidance of late-developing neurons in Caenorabditis elegans. Science 239: 643-645. 